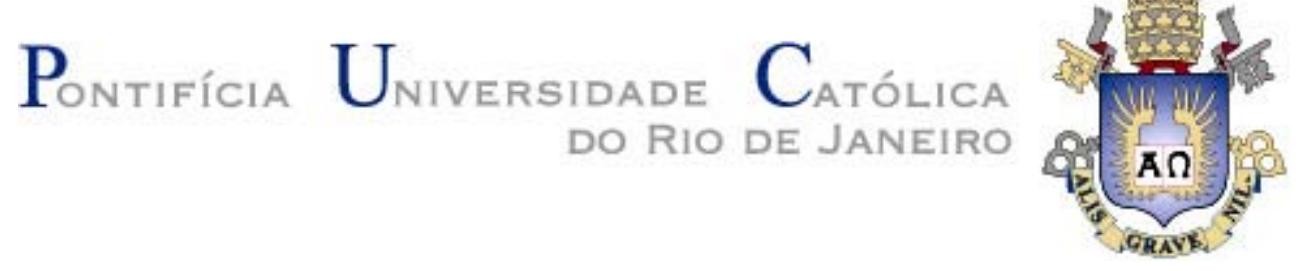

María Angélica Acosta Pérez

\title{
Estudo Numérico da Interação entre um Jato Supersônico e uma Superfície Plana.
}

Dissertação apresentada ao Programa de Pós-graduação em Engenharia Mecânica da PUC-Rio como requisito parcial para obtenção do título de Mestre em Engenharia Mecânica.

Orientador: Prof. Luis Fernando Figueira da Silva 


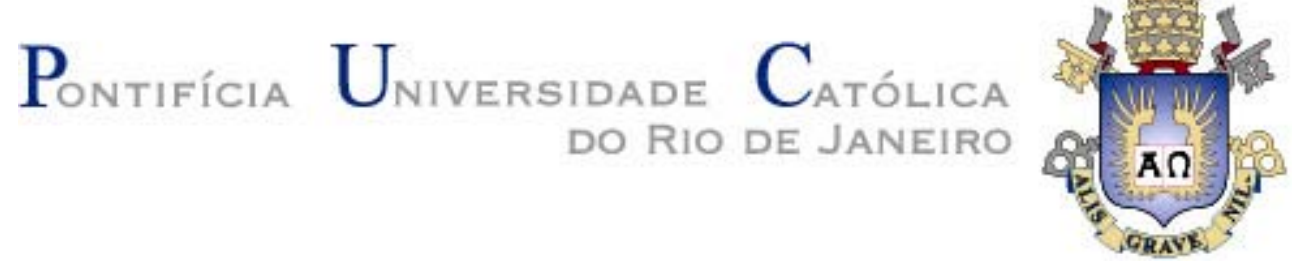

María Angélica Acosta Pérez

\section{Estudo Numérico a Interação entre um Jato Supersônico e uma Superfície Plana.}

Dissertação apresentada como requisito parcial para obtenção do grau de Mestre pelo Programa de Pós-graduação em Informática da PUC-Rio. Aprovada pela Comissão Examinadora abaixo assinada.

\section{Luis Fernando Figueira da Silva}

Orientador

Departamento de Engenharia Mecânica - PUC-Rio

Angela Ourivio Nieckele Departamento de Engenharia Mecânica - PUC-Rio

André Augusto Isnard

Departamento de Engenharia Mecânica - PUC-Rio 
Todos os direitos reservados. É proibida a reprodução total ou parcial do trabalho sem autorização da universidade, da autora e do orientador.

\section{María Angélica Acosta Pérez}

Formada em Engenharia Mecânica Universidad Francisco de Paula Santander - Colômbia em 2004.

Ficha Catalográfica

Acosta Pérez, María Angélica

Estudo numérico da interação entre um jato supersônico e uma superfície plana / María Angélica Acosta Pérez ; orientador: Luis Fernando Figueira da Silva. - 2008.

108 f. : il. ; $30 \mathrm{~cm}$

Dissertação (Mestrado em Engenharia Mecânica)-Pontifícia Universidade Católica do Rio de Janeiro, Rio de Janeiro, 2008.

Inclui bibliografia

1. Engenharia mecânica - Teses. 2. Métodos numéricos. 3. Volumes finitos. 4. Escoamentos compressíveis. I. Silva, Luis Fernando Figueira da. II. Pontifícia Universidade Católica do Rio de Janeiro. Departamento de Engenharia Mecânica. III. Título.

CDD: 621 
A Deus porque ele me deu coragem para permanecerde pé na luta. A minha família que são o motor da minha vida, a Roberth e a Maira pela amizade e pelo apoio nos momentos de fraqueza. 


\section{Agradecimentos}

Ao meu orientador, Professor Luis Fernando Figueira da Silva, pelo apoio, e orientação durante o desenvolvimento do curso de mestrado.

A toda minha família, em especial para minha mãe, pai, e irmão, pelo apoio de sempre.

Aos professores da PUC-Rio pelo ensino excelente, e pelos conhecimentos adquiridos.

Aos meus amigos e colegas do Laboratório de Termociências, com quem sempre compartilhei idéias, nos apoiamos nos estudos e em especial pela amizade.

Ao Departamento de Engenharia Mecânica da PUC-Rio e seus funcionários pela colaboração.

Finalmente minha gratidão à CAPES e à PUC-RIO pelos auxílios concedidos, sem os quais este trabalho não poderia ter sido possível. 


\section{Resumo}

Acosta Pérez, María Angélica; Figueira da Silva, Luis Fernando (Orientador). Estudo Numérico da interação entre um jato supersônico e uma superfície plana Rio de Janeiro 2008. 108p. Dissertação de Mestrado - Departamento de Engenharia Mecânica, Pontifícia Universidade Católica de Rio de Janeiro.

Neste trabalho é apresentado o estudo da interação entre um jato supersônico e uma superfície plana, com o objetivo de analisar o comportamento do campo de velocidade, pressão e temperatura do escoamento. Este estudo encontra sua motivação no processo de descamação térmica de rochas duras, a qual pode resultar da iteração entre um jato a alta pressão e temperatura e a rocha. Este processo, que pode ser útil na perfuração de rochas duras e profundas, ocorre devido ao acúmulo de tensões térmicas na rocha, o qual pode acarretar sua fratura. Este tipo de processo também envolve diversos mecanismos aerodinâmicos e termodinâmicos, que são isoladamente fenômenos abertos. No desenvolvimento deste trabalho o escoamento foi modelado pelas equações de Navier - Stokes bidimensionais para uma mistura de gases perfeitos em um sistema de coordenadas cilíndrico. O modelo considerado para descrever o transporte turbulento é o modelo de uma equação de Spalart - Allmaras, o qual envolve a solução de uma equação diferencial para a viscosidade turbulenta. Estas equações são resolvidas utilizando-se uma metodologia de volumes finitos adaptada a escoamentos compressíveis. A descrição dos escoamentos transientes obtidos necessitou de diversas modificações ao código computacional existente. Estas modificações trataram, em particular, das condições de contorno, que utilizam a noção de características, e do modelo de turbulência. A estrutura do escoamento resultante da interação entre o jato supersônico e a parede é estudada, avaliando-se a influência (i) da distância entre a saída do jato e a parede, (ii) da razão de pressões entre o jato e o ambiente. Além disso, é examinada a evolução transiente do escoamento. Os resultados obtidos são analisados com vista a obter as melhores condições aerodinâmicas para o processo de descamação térmica. 


\section{Palavras-chaves}

Métodos numéricos, volumes finitos, escoamento compressíveis.

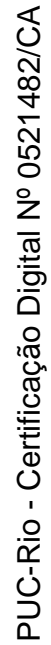




\section{Abstract}

Acosta Pérez, María Angélica; Figueira da Silva, Luis Fernando (Advisor). Numerical Study of the Interaction between a supersonic jet and planar surface Rio de Janeiro 2008. 108p. MSc. Dissertation Departamento de Engenharia Mecânica, Pontifícia Universidade Católica de Rio de Janeiro.

I in this work a study of the interaction between a supersonic jet and a planar surface is presented, with the aim to analyze the behavior of the velocity, pressure and temperature flowfield. This study finds its motivation in the process of thermal spallation of hard rocks, which may result from the interaction between a high pressure and high temperature jet and the rock. This process, that can be used in the drilling of hard and deep rocks, occurs due to the accumulation of thermal stresses in the rock, which can cause its fracture. This type of process also involves several aerodynamic and thermodynamic mechanisms, which are still open phenomena. In the development of this work the flow was modeled by the two-dimensional Navier-Stokes equation for a mixture of perfect gases in a cylindrical coordinates system. The model considered to describe the turbulent transport is the one equation of Spalart - Allmaras model, which involves the solution of a differential equation for the turbulent viscosity. These equations are solved using a finite volumes methodology which is adapted to compressible flows. The description of the obtained transient flow required several modifications in the existing computational code. These modifications involved, in particular, the choice of boundary conditions, that use the notion of characteristics, and the turbulence model. The structure of the flow resulting from the interaction between the supersonic jet and the wall is studied. In particular, are examined the influence (i) the distance between the jet and wall, (II) of the pressures ratio between the jet and the environment. Moreover, the transient evolution of the flow is examined. The obtained results are examined to determine the best aerodynamic conditions for the process of thermal spallation to occur. 


\section{keywords}

Numerical methods, finite volume, compressible flow.

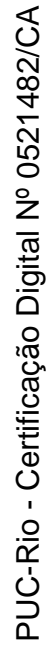




\section{Sumário}

1 Introdução 20

1.1. Objetivo do Trabalho 23

1.2. Organização do Trabalho 23

2 Conceitos Gerais e Revisão Bibliográfica 25

2.1. O Futuro da Energia Geotérmica 25

2.2. Técnicas de Perfuração Convencionais 27

2.3. Perfuração de Rochas por Jatos Quentes 32

2.4. Estudos Experimentais do Processo de Descamação Térmica 34

2.5. Mecanismos de Descamação Térmica 37

2.6. Modos de Fratura 39

2.7. Aerodinâmica 41

3 Formulação Matemática $\quad 44$

3.1. Equações Governantes 44

3.2. Modelo de Turbulência $\quad 49$

3.2.1. Modelo de uma Equação Diferencial de Spalart e Allmaras $\quad 49$

3.2.2. Extensões Propostas do Modelo de Turbulência de Spalart - Allmaras para Paredes Rugosas 53

3.3. Modelos do Fluxo de Calor em Presença de Descamação Térmica 56

3.4. Condições de Contorno 59

4 Método Numérico de Resolução 63

4.1. Método de Discretização Espacial 64

4.2. Método de Discretização Temporal $\quad 69$

4.3. Adaptação da Malha Computacional Procedimento de Refinamento e de $\begin{array}{ll}\text { Empobrecimento } & 70\end{array}$

4.4. Implementação de um esquema de movimentação de malha 71

5 Análise dos Resultados $\quad 75$

5.1. Seleção do Domínio Computacional 75

5.2. Estudo da Influência de $Z / D$ e de $p_{1} / p_{0}$ na Estrutura do Escoamento 77 
5.2.1. Resultados Obtidos para uma Razão de Pressão de $p_{1} / p_{0}=1$

5.2.2. Resultados Obtidos para uma Razão de Pressões $p_{1} / p_{0}=1,5$

5.3. Verificação do Modelo de Turbulência

6 Conclusões e Recomendações

94

6.1. Recomendações

7 Referências Bibliográficas

Anexo A 


\section{Lista de figuras}

Figura 1.1: Esquema do processo de descamação térmica (Wilkinson

e Tester, 1993b).

Figura 1.2: Profundidade de Perfuração Vs. Custos. (Wilkinson e Tester 1993).

Figura 2.1: Esquema do aproveitamento da energia geotérmica (Tester et al., 1994)

Figura 2.2: Broca de Perfuração Rotativa sem partes móveis "Broca de PCD (Diamante Policristalino Compactado)" (Bourgoyne et al, 1991).

Figura 2.3: Broca de Perfuração Rotativa sem partes móveis "Broca de Diamante Natural" (Bourgoyne et al, 1991).

Figura 2.4: Esquema de um offset das brocas tricônica (Bourgoyne et al, 1991).

Figura 2.5: Broca de Perfuração Rotativa com partes móveis "Broca Tricônica" (Site www.geocities.com.br).

Figura 2.6: Broca por Pulsos Percussivos "Broca Martelo" (Bourgoyne et al, 1991).

Figura 2.7: Perfuração de rocha por um jato quente (Rauenzahn, 1991).

Figura 2.8: Bancada para o desenvolvimento dos ensaios (Rodrigues et al, 2006)

Figura 2.9: Processo de Descamação. (Wilkinson e Tester, 1993).

Figura 2.10: Escoamento compressível supersônico e subsônico não estacionário e turbulento.

Figura 2.11: Transferência de calor entre os gases quentes e a superfície da rocha por convecção e por radiação.

Figura 2.12: Interação entre as escamas sólidas oriundas da rocha e o escoamento.

Figura 3.1: Domínio computacional e condições de contorno

Figura 4.1: Esquema representativo da disposição do volume "i"

Figura 4.2 Esquema da técnica de empobrecimento de malha (Walter et al, i.e, 2005).

Figura 4.3 Triângulos que determinam o lado $i k$. 
Figura 4.4 Malha utilizada para a validação do deslocamento desta. (a) Malha original, (b) Malha movimentada após de $1 / 4$ de período.

Figura 5.1: Malha 1, tentativa de se modelar apenas as linhas de corrente.

Figura 5.2: Malha 2, incluindo o bocal, com fronteira de saída próxima deste.

Figura 5.3: Malha 3, incluindo o bocal, com fronteira de saída distante deste.

Figura 5.4: Malha utilizada para a análise da influência de $Z / D$, (a)

$$
Z / D=15 \text {; (b) } Z / D=20 \text {. }
$$

Figura 5.5: Evolução temporal do logaritmo do resíduo da densidade, para um razão de $p_{1} / p_{0}=1$ e diferentes distâncias entre a saída do bocal e a superfície.

Figura 5.6: Evolução da pressão estática (atm) no escoamento para $Z / D=15$ (a) vista englobando o bocal e a parede (b) na vizinhança da parede, distância em (cm).

Figura 5.7: Evolução da pressão estática (atm) no escoamento para $Z / D=20$, distância em (cm).

Figura 5.8: Evolução do número de Mach no escoamento para $Z / D=15$, distância em (cm).

Figura 5.9: Evolução do número de Mach no escoamento para $Z / D=20$, distância em (cm).

Figura 5.10: Pressão (atm) e Temperatura $(\mathrm{K})$ para $Z / D=20$ e $p_{1} / p_{0}=1$ distância em (cm).

Figura 5.11: Evolução logarítmica do resíduo da densidade para uma razão de pressão de $p_{1} / p_{0}=1.5$ e diferentes relações de $Z / D$.

Figura 5.12: Evolução da pressão estática no escoamento e na vizinhança da parede; e do número de Mach para $Z / D=15$ e $p_{1} / p_{0}=1,5$ distância em (cm).

Figura 5.13: Evolução da pressão estática e do número de Mach no escoamento para $Z / D=20$ e $p_{1} / p_{0}=1,5$ distância em 
(cm).

Figura 5.14: Evolução da pressão ao longo da linha de simetria para $Z / D=20$.

Figura 5.15: Domínio computacional utilizado para a avaliação do programa em situações de camada limite desenvolvendose em numa superfície plana, (a) vista geral (b) detalhe da camada limite na vizinhança da parede, (c) direção do escoamento.

Figura 5.16 Vetor velocidade e camada limite sobre uma superfície plana adiabática para um escoamento supersônico, (a) vista geral, (b) detalhe do escoamento na vizinhança da parede.

Figura 5.17: Evolução logarítmica do resíduo da densidade no caso do escoamento com camada limite.

Figura 5.18: Evolução transversal da velocidade $(\mathrm{cm} / \mathrm{s})$ e da viscosidade turbulenta $(n)$ modificada para o número de Reynolds $10^{4}$.

Figura 5.19: Evolução longitudinal da temperatura estática (K) a uma distância de 2,5 (cm) para um escoamento supersônico. 


\section{Lista de tabelas}

Tabela 5.1 Valores da relação $Z / D$ utilizados.

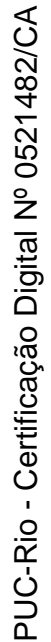




\section{Lista de símbolos}

\begin{tabular}{|c|c|c|}
\hline & $a$ & Velocidade do som \\
\hline & $B$ & Vetor das variáveis conservadas \\
\hline & $C_{b 2}$ & Constante empírica \\
\hline & $C_{L}$ & Fator de forma das escamas \\
\hline & $C p$ & Calor específico a pressão constante \\
\hline & $C\left(Q_{i}\right)$ & Operador convectivo \\
\hline & $C v$ & Calor específico a volume constante \\
\hline & $C_{v 1}$ & Constante empírica \\
\hline & $d$ & Distância à parede mais próxima \\
\hline & $D$ & Diâmetro da saída do bocal \\
\hline & $E$ & Energia total específica \\
\hline 象 & $e$ & Energia interna específica \\
\hline ్ㅠㅇ & $F, G$ & Vetores de fluxo nas direções longitudinal, $x$, e radial, $y$ \\
\hline$\frac{z}{\frac{z}{\sigma}}$ & $f_{v 1}$ & Amortecimento viscoso \\
\hline $\begin{array}{l}\overline{0} \\
\text { 㯊 }\end{array}$ & $G_{v}$ & Taxa de produção da viscosidade turbulenta modificada \\
\hline Tु & $H$ & Entalpia total específica \\
\hline$c^{c}$ & $h$ & Entalpia específica \\
\hline$\frac{\circ}{\frac{1}{x}}$ & $I$ & Parâmetro de controle \\
\hline & $J$ & Meio período de oscilação \\
\hline & K & Vetor dos termos de produção \\
\hline & $l$ & Comprimento \\
\hline & $l_{0}$ & Comprimento característico \\
\hline & $m$ & Parâmetro de homogeneidade da rocha \\
\hline & $M$ & Número de Mach \\
\hline & $N$ & Número de iterações \\
\hline & $n_{x}, n_{y}$ & Componentes do vetor unitário normal à face $i k$ \\
\hline & $\mathrm{P}$ & Probabilidade \\
\hline & $p$ & Pressão estática \\
\hline & $\operatorname{Pr}$ & Número de Prandtl \\
\hline
\end{tabular}




\begin{tabular}{|c|c|}
\hline$Q_{i}^{n}$ & Solução no passo de tempo $n$ \\
\hline$\dot{Q}$ & Fluxo de calor \\
\hline $\operatorname{Re}$ & Número de Reynolds acústico \\
\hline$p_{1} / p_{0}$ & Razão de pressões \\
\hline$R_{\text {noz }}$ & Raio da saída de bocal do jato \\
\hline$\tilde{S}$ & Tensor vorticidade \\
\hline$S_{f}$ & Operador do termo corretivo \\
\hline$S_{Q}$ & Operador correspondente à integração do termo químico \\
\hline St & Número de Stanton \\
\hline$t$ & Tempo \\
\hline$T$ & Temperatura estática \\
\hline$T_{\text {ro }}$ & Temperatura inicial da rocha no estado de equilíbrio \\
\hline$T_{s}$ & Temperatura da rocha no momento da descamação \\
\hline$U$ & Velocidade \\
\hline$U_{x k}$ & Velocidade da difusão da espécie $k$ na direção $x$ \\
\hline$U_{y k}$ & Velocidade da difusão da espécie $k$ na direção $y$ \\
\hline$u_{t}$ & Velocidade de atrito \\
\hline$u$ & Velocidade na componente $x$ \\
\hline$v$ & Velocidade na componente $y$ \\
\hline$V$ & Volume \\
\hline$\dot{w}$ & Taxa de produção molar \\
\hline$W$ & Massa molar \\
\hline$x, y$ & Direções do sistema cartesiano de coordenadas \\
\hline$Y_{i}$ & Fração de massa \\
\hline$Z$ & Distância entre a saída do bocal e a parede \\
\hline
\end{tabular}

\section{$\underline{\text { Símbolos Gregos }}$}

$\alpha \quad$ Difusividade térmica

$\beta \quad$ Coeficiente linear de expansão térmica 


$\begin{array}{ll}\mathrm{E} & \text { Modulo de Young } \\ \gamma & \text { Razão de calores específicos } \\ \lambda & \text { Condutividade } \\ \mathrm{K} & \text { Constante de Von Kármán } \\ \mu & \text { Viscosidade da mistura } \\ \mu_{t} & \text { Viscosidade turbulenta modificada } \\ \Omega & \text { Tensor taxa de rotação média } \\ \rho & \text { Densidade } \\ \sigma & \text { Tensão de ruptura } \\ \tau & \text { Tensão devido à viscosidade } \\ \theta & \text { Ângulo inicial da rampa } \\ \delta & \text { Deslocamento } \\ v & \text { Coeficiente de Poisson } \\ \Re & \text { Constante universal dos gases } \\ \xi_{v} & \text { Constante empírica } \\ \psi_{v} & \text { Taxa de distribuição da viscosidade turbulenta modificada }\end{array}$

\section{Subscritos}

$\begin{array}{ll}i, j & \text { Índices } \\ i k & \text { Índice relativa a face } i k \\ j e t & \text { Gases de saída do jato } \\ L & \text { Direção à esquerda da face } \\ n & \text { Direção normal } \\ R & \text { Direção à direita da face } \\ r & \text { Rocha }\end{array}$

\section{Sobrescritos}




\section{LISTA DE SIGLAS E ABREVIATURAS}

HDR

MIT

SOD

PCD

INPE

LCP

DEM

PUC - Rio
Rochas duras quentes "Hot Dry Rock"

Massachusetts Institute of Technology

Distância de afastamento "Stand off Distance"

Diamante Policristalino Compactado

Instituto Nacional de Pesquisas Espaciais

Laboratório Associado de Combustão e Propulsão

Departamento de Engenharia Mecânica

Pontifícia Universidade Católica do Rio de Janeiro 